\title{
Biomimetic Sensor Suite for Flight Control of a Micromechanical Flight Insect: Design and Experimental Results *
}

\author{
W.C. Wu L. Schenato R.J. Wood R.S. Fearing \\ Department of EECS, University of California, Berkeley, CA 94720 \\ $\{w c w u$, lusche, rjwood, ronf\}@robotics.eecs.berkeley.edu
}

\begin{abstract}
Four types of biomimetic sensors have been designed and simulated for flight control of a robotic flying insect. The ocelli use four photodiodes to detect changes in light intensity in the surrounding. The halteres use piezo-actuated vibrating structures to sense the Coriolis forces to detect angular velocities. The optic flow sensors consist of linear arrays of elementary motion detectors to register optic flows. The MEMS compass uses three metal loops to detect changes in the magnetic field. Despite simplicity and novelty, the preliminary tests on these devices showed promising performances for using such biomimetic sensors on a robotic flying insect.
\end{abstract}

\section{Introduction}

Micro aerial vehicles (MAVs) have drawn a great deal of attention in the past decade due to the quick advances in microtechnology and several groups have worked on MAVs based on fixed and rotary wings [5]. However, flapping flight provides superior maneuverability that would be beneficial in obstacle avoidance and for navigation in small spaces. The UC Berkeley Micromechanical Flying Insect (MFI) project uses biomimetic principles to develop an inch-size, flappingwinged robot that will be capable of sustained autonomous flight [2][14].

The MFI will be equipped with different types of sensors that are important for stabilizing flight as well as navigation. Because of the limited size and power budget available to the MFI, the designs (package size, power requirements, etc.) of commercially available micro sensors are in general not suitable for the MFI. On the other hand, novel biomimetic devices based on the sensory systems of real insects are considered. In particular, ocelli, halteres, optic flow sensors, and MEMS magnetic compass have been designed and implemented. The ocelli are used to estimate body attitude relative to a fixed frame. The halteres are used

${ }^{*}$ This work was funded by NSF KDI ECS 9873474, ONR MURI N00014-98-1-0671, and DARPA. to measure body rotational velocities. The optic flow sensors are used to avoid objects in the flight course as well as for stabilization. The MEMS compass is used to adjust an insect's heading. These devices have the virtues of simple design, easy implementation, low power consumption, and high performance. This paper presents the designs, simulations, and experimental results of these biologically inspired sensing devices.

\section{Ocelli}

The ocelli are sensory organs present in many flying insects. This system consists of three wide angle photoreceptors on the head of an insect. They are oriented in such a way that they collect light from different regions of the sky (see Figure 1). Albeit the exact physiology of the ocelli and their scope in insect flight are still not completely unveiled, it is believed that they play a fundamental role in attitude stabilization, in particular, in horizon stabilization [4][10].

Biologists believe that ocelli estimate the orientation of the insect with respect to the sky by comparing the intensity of light measured by the different photoreceptors. Their argument is based on the assumption that, as a first approximation, the intensity of light, $I$, measured by the photoreceptors is only a function of its latitude relative to the light source (i.e. the sun). In our implementation, we use four photodiodes for the ocelli system. Although real insects have three ocelli, we prefer a four-receptor configuration because the design is simplified and the results are intuitive. Also, our concept can be easily extended to the three-receptor case.

Four ideal photoreceptors, $P_{1}, P_{2}, P_{3}, P_{4}$, are fixed with respect to the body frame, $B$. They are oriented symmetrically such that they have the same latitude and their axes intersect the sky sphere forming an imaginary pyramid whose vertex is placed at the center of the insect head. Formally, their orientations relative to the body frame, $B$, can be represented in 


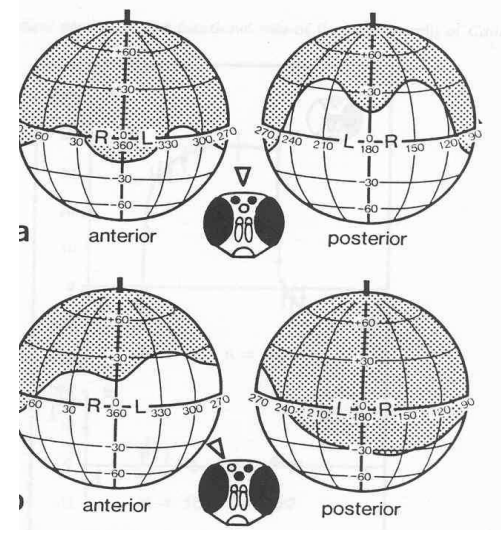

Figure 1: Head of a blowfly and the fields of reception of its ocelli (curtesy of [10]).

Cartesian coordinates as follows:

$$
\begin{aligned}
& P_{1}^{b}=\left[\begin{array}{lll}
\sin \alpha & 0 & \cos \alpha
\end{array}\right]^{T}, \quad P_{2}^{b}=\left[\begin{array}{lll}
-\sin \alpha & 0 & \cos \alpha
\end{array}\right]^{T} \\
& P_{3}^{b}=\left[\begin{array}{lll}
0 & \sin \alpha \cos \alpha
\end{array}\right]^{T}, \quad P_{4}^{b}=\left[\begin{array}{lll}
0 & -\sin \alpha \cos \alpha
\end{array}\right]^{T}
\end{aligned}
$$

where the parameter, $\alpha \in(0, \pi]$, sets the latitude of the photoreceptors. Each photoreceptor collects light from a conic region, $A_{i}$, of the sky around its orientation $P_{i}$ as shown in Figure 2.

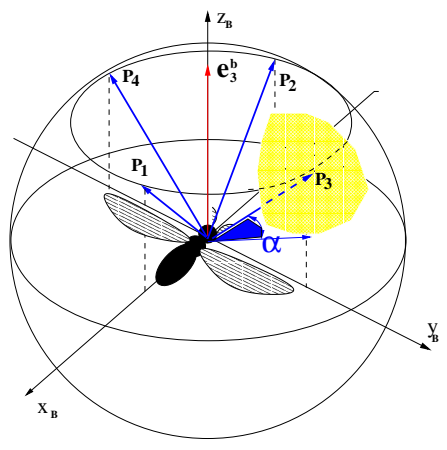

Figure 2: Graphical rendering of ocelli. Four photoreceptors, $P_{1}, P_{2}, P_{3}, P_{4}$, collect light from different regions of the sky. The shadowed area represents the receptive region of photoreceptor $P_{3}$.

The measurements from the photoreceptors are simply subtracted pairwise and these two signals are the output from the ocelli:

$$
\begin{aligned}
& y_{1}=I\left(P_{1}\right)-I\left(P_{2}\right) \\
& y_{2}=I\left(P_{3}\right)-I\left(P_{4}\right)
\end{aligned}
$$

where $I\left(P_{i}\right)$ is the output from the $i^{t h}$ photodiode. If the output of a photodiode is a monotonically decreas- ing function of its latitude relative to the light source, we have the following proposition:

Proposition 1. If the photoreceptor output is a monotonically decreasing function of its latitude, $\theta_{i}$, relative to the light source, then the signals, $y_{1}$ and $y_{2}$, defined in Equations (2) always satisfy the following conditions:

$$
\begin{aligned}
& k_{\min } S_{x} \leq y_{1} \leq k_{\max } S_{x} \\
& k_{\min } S_{y} \leq y_{2} \leq k_{\max } S_{y}
\end{aligned}
$$

where $0<k_{\min }<k_{\max }$ are constants, and $S_{x}$ and $S_{y}$ are, respectively, the $x$ and $y$ projections of the light source on the $x-y$ plane of the ocelli.

It is evident that the output from the ocelli can be used as an estimate of the position of the ocelli reference frame relative to the light source. Thus, they can be used to align the ocelli reference frame with the light source as described in detail in [9].
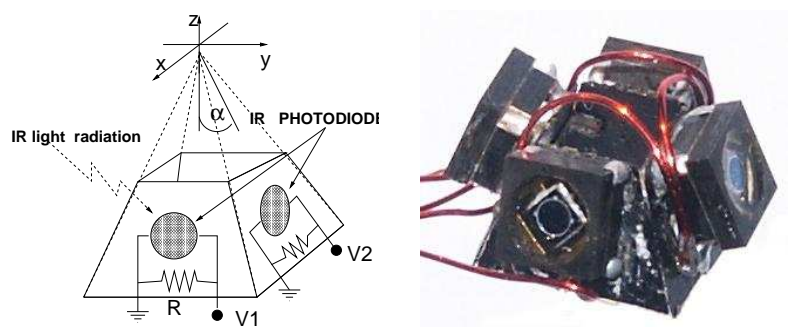

Figure 3: (a) Schematic of ocelli design; (b) Photo of the ocelli structure.

Based on the mathematical modeling, we have designed a biomimetic ocelli system. The device has four IR photodiodes soldered onto a pyramid as shown in Figure 3. Each photodiode collects light radiation, which induces electric current that is proportional to the intensity of light collected and the active area of the photodiode. Each photodiode is placed in series with a small resistor and the voltage drop across the resister is measured. The output voltages from the four photodiodes are combined differentially to give the two ocelli outputs, which are used as estimators of light source position:

$$
\begin{aligned}
& y_{1}=V_{1}-V_{3} \\
& y_{2}=V_{2}-V 4
\end{aligned}
$$

To test sensitivity and output range of the ocelli sensors, the light source is positioned at $P_{S}=(0,0, h)$, where $h$ is the height from the plane of the ocelli to the light source. The orientation of the pyramid is kept constant such that the photodiodes 1 and 3 are parallel to the $x$-axis, while 2 and 4 are parallel to the $y$-axis. Then, the ocelli are moved to different $x-y$ 
positions and the recorded output is shown in Figure 4. The ocelli output gives an excellent estimate of the distance from the ocelli structure to the origin $O=(0,0,0)$. The pyramid with angle $\alpha=40^{\circ}$ shows the best performance in terms of range and linearity with distance. The measurements did not need any kind of noise filtering and the results were repeatable. The size of the whole structure is about $5 x 5 x 5 \mathrm{~mm}$ and it weighs $150 \mathrm{mg}$. However, the weight can be further reduced if bare photodiodes are used since the active area of one photodiode is less than $1 \mathrm{~mm}^{2}$.
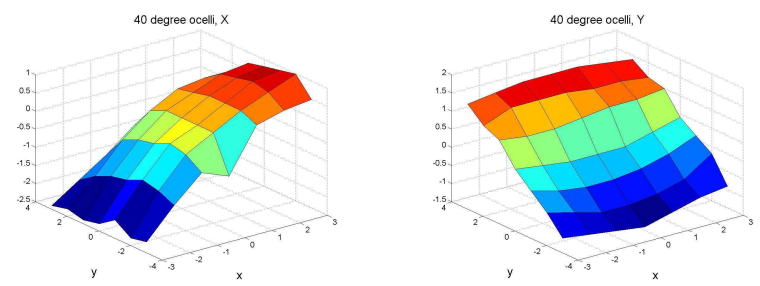

Figure 4: $y_{1}$ (left) and $y_{2}$ (right) measurements for ocelli structure with $\alpha=40^{\circ}$.

\section{$3 \quad$ Haltere}

Research on insect flight revealed that insects use structures, called halteres, to measure body rotations via gyroscopic forces [3]. The halteres of a fly resemble small balls at the end of thin sticks. During flight the two halteres beat up and down in non-coplanar planes through an angle of nearly $180^{\circ}$ anti-phase to the wings at the wingbeat frequency. This non-coplanarity of the two halteres is essential for a fly to detect rotations about all three turning axes [6].

As a result of insect motion and haltere kinematics, a complex force acts on the halteres during flight. Among the inertial, angular acceleration, centrifugal, Coriolis, and gravitational components, only the Coriolis forces contain useful information on the axis, sign, and magnitude of the insect's body rotation. In order to retrieve the Coriolis component, the force signals orthogonal to a haltere's beating plane are measured because all other interfering force components are small in this direction. Furthermore, because the Coriolis forces are dependent on the haltere velocity, these signals are modulated in time with the haltere beat frequency. Thus, utilizing the characteristics (frequency, modulation, and phase) of the Coriolis signals on the left and right halteres, a demodulation scheme has been proposed to decipher roll, pitch, and yaw rotations [13]. For simulations, the angular velocities of an insect under hovering condition are generated by the Virtual Insect Flight Simulator (VIFS) [8]. The results are shown in Figure 5.
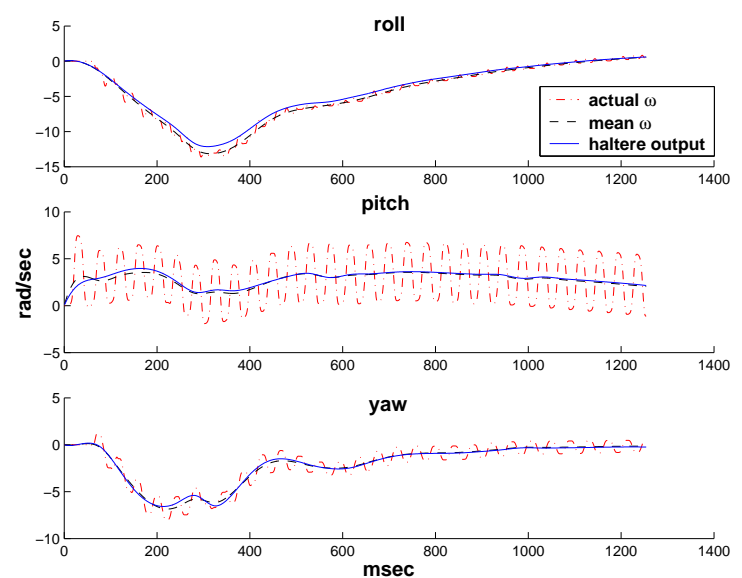

Figure 5: Angular rotation detection by halteres.

Since a haltere must have only one sensing degree of freedom (i.e. the direction orthogonal to the haltere beating plane), the design of a mechanical haltere must allow for high stiffness in the tangential direction and compliance in the lateral direction. The best case mechanically for this is a flat beam with the wide face in the plane of the haltere beating and the end of the beam is twisted to allow a high $Q$ compliant section for rotation as in Figure 6a. To detect the Coriolis forces, two strain gages are placed, one on either side of the beam, close to the point of rotation such that one would be in compression while the other is in tension. In addition, because the Coriolis forces are proportional to the haltere velocity, it is desired to have a high haltere beat frequency and a large stroke. This can be achieved by placing the haltere on the output link of a fourbar mechanism driven by a piezoelectric actuator, similar to the method used to drive the MFI wing as described in [2][14]. Figure $6 \mathrm{~b}$ shows the completed haltere.

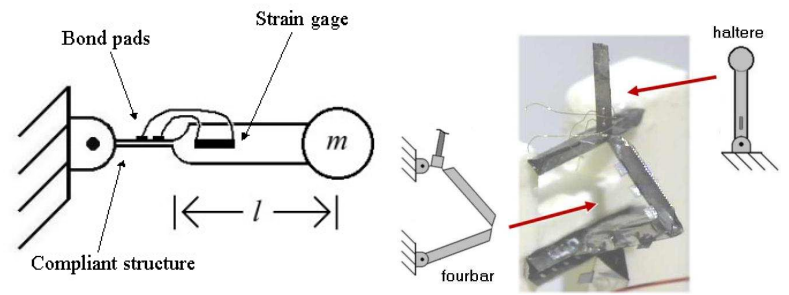

Figure 6: (a) Haltere design parameters; (b) Photo of the completed haltere. Adapted from [13].

The test results for the haltere structure are seen in Figure 7. With the fourbar driven structure, the position of the haltere can be sensed using actuatormounted strain sensors as described in [12]. This hal- 
tere position is normalized to yield a unity magnitude sine wave which represents the haltere phase. This is then used to demodulate the force signals using the proposed demodulation scheme. The performance of the haltere shows some key features for use on the MFI. First, the haltere needs very little power since it can be driven parasitically from the body vibrations of the MFI. Second, the haltere has a large dynamical range to accommodate slow turns as well as saccades. Finally, when the wings of the MFI are flapping, the wing inertia will cause the MFI body to oscillate along the axis perpendicular to the stroke plane. The haltere can reduce the error caused by these oscillations by phase-locking to the wing.
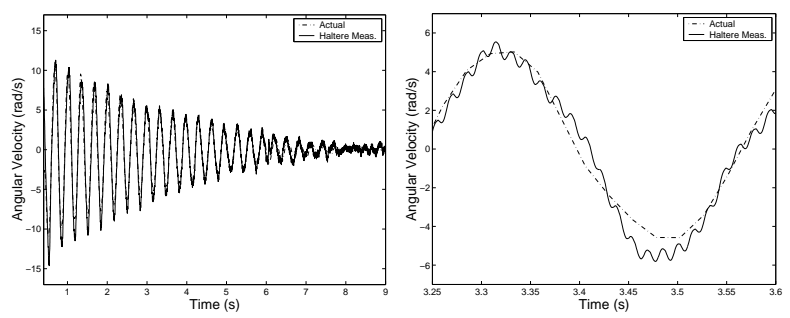

Figure 7: (a) Result for the fourbar actuated haltere; (b) Zoomed in to show accuracy. Adapted from [13].

\section{Optic Flow Sensor}

Studies of insect's visual behaviors contributed to the well-characterized optomotor response. When a fly is presented with a leftward (or rightward) moving stimulus, it would turn in the direction of stimulus movement in order to reduce the image motion on its eyes. Such a response is believed to help the fly maintain a straight course by compensating for undesired deviations [11]. Hassenstein and Reichardt proposed a motion detection mechanism using the spatiotemperal elements of image motion [7]. This type of correlationbased algorithm is excellent at explaining the visual processing pathway underlying behaviors such as the optomotor response.

The building block of the Reichardt motion sensor is an elementary motion detector (EMD) whose structure is shown in Figure 8a. When a moving image is presented to an EMD, the perceived signal in one receptor is compared to the delayed signal in a neighboring receptor. If the left signal correlates more strongly to the delayed right signal, then the image is moving from right to left and vice versa. In the EMD implementation, the bandpass filter represents the temporal frequency response of the photoreceptor where the DC and high frequency components of illumination are eliminated. The first order lowpass filter provides the delay operation and the multipli- cation achieves the correlation required by the EMD. The opponent subtraction results in different signs for the leftward and rightward image motions. Because an EMD can not detect image motion that is perpendicular to the transverse axis of the two receptors, two EMDs in a cross configuration are used so that it is possible to detect image motion in all directions. Figure $8 \mathrm{~b}$ shows the completed structure of the EMDs.

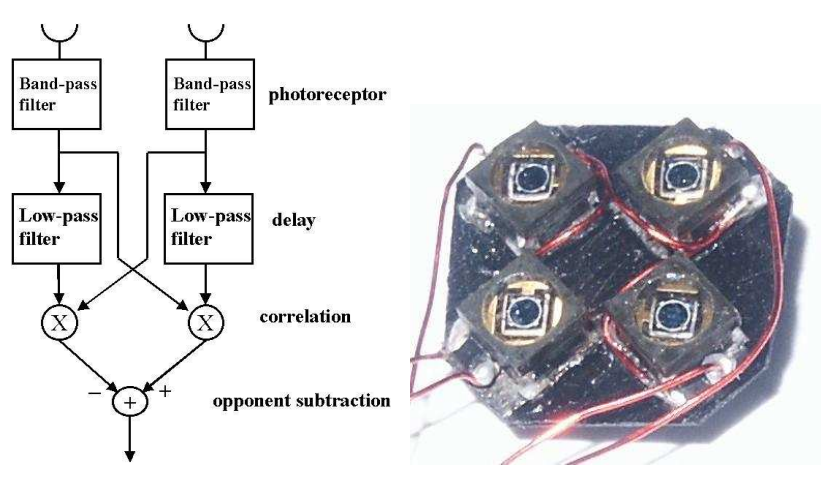

Figure 8: (a) Elementary motion detector architecture; (b) Photo of two EMDs oriented in orthogonal directions.

Although a device consisting of only two photodiodes can hardly be called a motion sensor, the purpose of our device is to detect optic flows induced by objects when the MFI is moving. To test our optic flow sensor, we shined IR light on a piece of white paper with a black stripe on it. Then, we slowly moved the sensor across the paper and recorded the outputs from both photodiodes. The results are shown in Figure 9a. The outputs of these two diodes are further processed by the delay-and-correlate operation and the result is given in Figure 9b. It is obvious that with only two photodiodes, our device is still able to register optic flows.
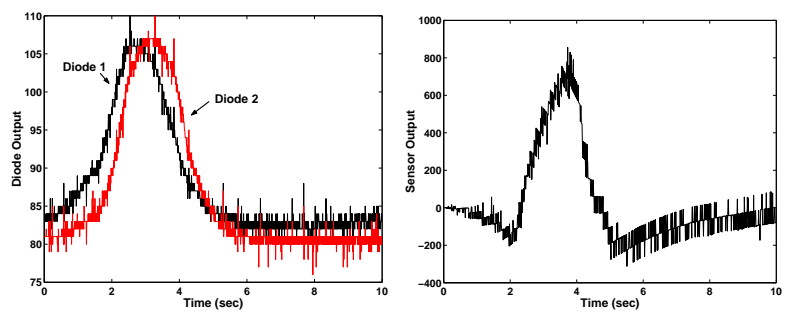

Figure 9: (a) Output from photodiodes 1 and 2; (b) One DOF EMD output.

Optic flows perceived by a flow sensor can be exploited to achieve tasks such as obstacle avoidance and terrain tracking. In the simulation, a 1D flow sensor consisting of an array of EMDs is used for a fly to fol- 
low a simple topography of the ground. The top panel in Figure 10 illustrates the simulation setup. An object on a uniform ground is in the flight course of a fly. A flow sensor is placed on the head of the fly. The bottom panel shows the optic flows perceived by the sensor during the flight. When the fly approaches the object, the optic flows increase dramatically. By setting an upper threshold for the flows, the fly would elevate when this value is reached in order to avoid hitting the object or to maintain a safe distance to the object. Being at a higher position, the distance from the fly to the object increases and hence the optic flows drop. After the fly passes the object, the flows decrease even faster because the ground does not induce optic flows due to its uniformity. At this point, the fly would descend only when a preset lower threshold is reached.



Figure 10: Simple terrain tracking using a 1D flow sensor. Dashed line: instantaneous optic flow. Solid line: accumulated flow.

Although both the instantaneous and the accumulated optic flows show similar trends, the accumulated flow is a preferred signal since its magnitude varies more drastically and hence it would be easier to choose appropriate upper and lower thresholds. In the simulation, a leaky integrator for the accumulated signal is used to emphasize the recent events over the old events. Thus, by adjusting three parameters (upper and lower threshold values and the forgetting factor of the leaky integrator), desired terrain tracking can be achieved.

\section{MEMS Magnetic Compass}

Attitude control of MFI body requires a set of sensors that can estimate its orientation relative to a fixed frame. The ocelli system provides a means to reorient the insect body towards a specific direction, however, the insect's heading remains arbitrary. Since heading is important for forward flight and maneuvering, we propose using a MEMS magnetic compass for the MFI. This magnetic sensor can estimate the heading based on the terrestrial geomagnetic field. The MEMS compass is a U-shaped suspended structure (see Figure 11a), similar to that proposed in [1]. Electric current flows through this structure, interacting with the terrestrial geomagnetic field, and induces the Lorentz force:

$$
F=L I B \sin \alpha
$$

where $F$ is the total force at the tip of the cantilever, $L$ is the length of one side of the cantilever, $I$ is the total current, $B$ is the terrestrial electromagnetic field, and $\alpha$ is the angle between the direction of the magnetic field and the electric current. The deflection of the cantilever, which is proportional to the force perpendicular to the cantilever, is sensed at the base by strain gages whose output can be used to estimate the heading of the MFI. Given the stringent requirements imposed by the MFI design, this sensor needs to have a small size $L<20 \mathrm{~mm}$, a sensitivity $\delta \alpha<1^{\circ}$ for $-60^{\circ}<\alpha<60^{\circ}$, a large bandwidth $f>2 k H z$, and small power consumption $P<2 m W$. These requirements depend on the geometric design variables as follows:

$$
\begin{aligned}
\delta \alpha & =\frac{180 E w t^{2} \epsilon_{\min }}{9 \pi B L^{2} I} \\
f & =\frac{1}{4 \pi L} \sqrt{\frac{3 E t}{14 \rho}} \\
P & =\frac{3 I^{2} \varrho L}{w t}
\end{aligned}
$$

where $E$ is the Young's modulus of stainless steel, $w$ is the width of the cantilever beam, $t$ is the thickness, $\epsilon_{\text {min }}$ is the minimum sensitivity of the strain gage, $\rho$ is the density of stainless steel, and $\varrho$ is the resistivity of stainless steel. Since there are several performance metrics that can be chosen, we tried to optimize the sensor sensitivity while satisfying the constraints on size, power consumption and bandwidth. Moreover, electric current and beam thickness were fixed, while cantilever width and length were the design variables. Figure 12 shows performance variables as a function of cantilever width and length. Table 1 shows the optimal width and length and the corresponding performance in terms of sensitivity, power consumption, and bandwidth. These data are promising since they satisfy the stringent requirements imposed by the MFI design, while provide a simple way to estimate the heading of the MFI. A prototype MEMS compass has been built (see Figure 11b).

\section{Conclusions and Future Work}

In this paper we presented the sensory system for the MFI. It consists of four biomimetic devices: ocelli, haltere, optic flow sensor, and magnetic sensor, which are essential for the MFI to maintain stable flight as well as achieve simple maneuvering. Although high precision micro sensors are available, they generally do not meet the stringent requirements of MAVs as small as the MFI. The design of our devices has taken 

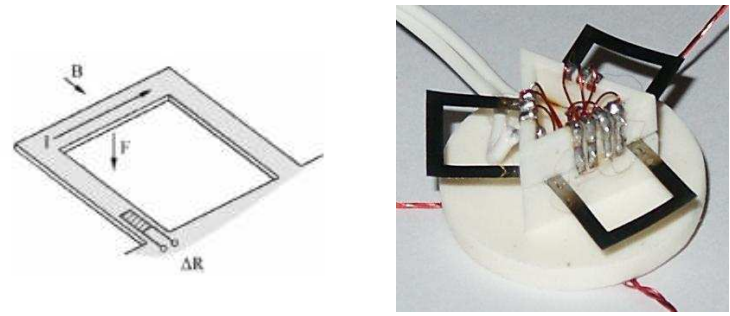

Figure 11: (a) Schematic of MEMS compass design; (b) Photo of the magnetic sensor with three loops.

\begin{tabular}{|c|c|c|}
\hline Parameter & Value & Unit \\
\hline$\rho$ & 8000 & $K g / m^{3}$ \\
$\epsilon_{\min }$ & $10^{-7}$ & \\
$E$ & 193 & $G P a$ \\
$I$ & 1 & $m A$ \\
$t$ & 5 & $\mu m$ \\
$\varrho$ & 321 & $\Omega / m$ \\
$B$ & 50 & $\mu T$ \\
\hline$L$ & 13 & $m m$ \\
$w$ & 160 & $\mu m$ \\
$f$ & 4.4 & $k H z$ \\
$\delta \alpha$ & 1 & $d e g$ \\
$P$ & 1.45 & $m W$ \\
\hline
\end{tabular}

Table 1: Fixed parameters are on top and optimized variables are at bottom.

into account the size, power budge, and computational power of the MFI while still be able to show high performances. Moreover, our devices can be further improved without significant revisions of their structures. In the future, these sensors will be integrated to the flight mill, an apparatus that demonstrates simplified aerodynamics of flapping flight, in order to investigate their performances as a whole sensory system and test different flight control techniques using output feedback from this sensory system.

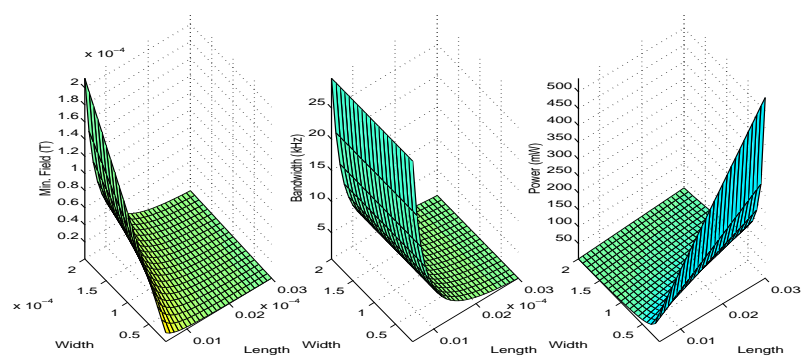

Figure 12: Minimum detectable magnetic field, bandwidth and power dissipation of the magnetic sensor as a function of length and width of cantilever.

\section{References}

[1] V. Beroulle, Y. Bertrand, L. Latorre, and P. Nouet. Test and testability of a monolithic mems for magnetic field sensing. Journal of Electronic Testing: Theory and Applications, 17:439-50, 2001.

[2] R.S. Fearing, K.H. Chiang, M.H. Dickinson, D.L. Pick, M. Sitti, and J. Yan. Wing transmission for a micromechanical flying insect. In Proc of the IEEE Int'l Conf on Robotics and Automation, pages 15091516, San Francisco, CA, April 2000.

[3] R. Hengstenberg. Mechanosensory control of compensatory head roll during flight in the blowfly Calliphora erythrocephala Meig. Journal of Comparative Physiology A, 163:151-165, 1988.

[4] RG Kastberger. The ocelli control the flight course in honeybees. Physiological Entomology, 15:337-346, 1990.

[5] B. Motazed, D. Vos, and M. Drela. Aerodymatics and flight control design for hovering MAVs. In Proc of Amer Control Conference, Philadelphia, PA, June 1998.

[6] G. Nalbach. The halteres of the blowfly Calliphora: I. kinematics and dynamics. Journal of Comparative Physiology A, 173:293-300, 1993.

[7] W. Reichardt. Autocorrelation, a principle for relative movement discrimination by the central nervous system. MIT Press, New York, 1961.

[8] L. Schenato, X. Deng, W.C. Wu, and S. Sastry. Virtual insect flight simulator (VIFS): a softwar testbed for insect flight. In Proc of the IEEE Int'l Conf on Robotics and Automation, pages 3885-3892, Seoul, South Korea, May 2001.

[9] L. Schenato, W.C. Wu, and S. Sastry. Attitude control for a micromechanical flying insect via sensor output feedback. In The 7th Int'l Conf on Control, Automation, Robotic and Vision, Singapore, Dec 2002.

[10] H. Schuppe and R. Hengstenberg. Optical properties of the ocelli of calliphora erythrocephala and their role in the dorsal light response. Journal of Comparative Biology A, 173:143-149, 1993.

[11] M.V. Srinivasan, M. Poteser, and K. Kral. Motion detection in insect orientation and navigation. Vision Research, 39:2749-2766, 1999.

[12] R.J. Wood and R.S. Fearing. Flight force measurement for a micromechanical flying insect. In Intelligent Robots and Systems, Maui, HI, Oct 29-Nov 3 2001.

[13] W.C. Wu, R.J. Wood, and R.S. Fearing. Halteres for the micromechanical flying insect. In Proc of the IEEE Int'l Conf on Robotics and Automation, Washington, DC, May 2002.

[14] J. Yan, R.J. Wood, S. Avadhanula, R.S. Fearing, and M. Sitti. Towards flapping wing control for a micromechanical flying insect. In Proc of the IEEE Int'l Conf on Robotics and Automation, pages 3901-3908, Seoul, South Korea, May 2001. 